\title{
Detection of meteorological inconsistencies by GPS
}

\author{
Lorenzo Balestri, Mauro Boccolari, Slobodan Fazlagić, Sergio Pugnaghi and Renato Santangelo \\ Dipartimento Ingegneria dei Materiali e dell'Ambiente (Osservatorio Geofisico), \\ Università degli Studi di Modena e Reggio Emilia, Modena, Italy
}

\begin{abstract}
GPS observations, distances from satellites to receivers and meteorological conditions in neutral atmosphere are known to obey a constraint, which provides a residual or in other words a quality index. A method is discussed which provides a residual epoch by epoch in near real time. In general, distribution of residuals during several consecutive epochs belonging to the same satellites, allows estimates of a mean and a standard deviation of mean. Under normal meteorological conditions distribution of residuals appears to be consistent with zero mean as expected. However, consecutive residuals sometimes appear to have a mean different from zero by more than three standard deviations of mean. Such significant consecutive epochs provide a warning of existing inconsistencies among GPS observations, distances from satellites to receivers as obtained by orbital information, meteorological conditions above receivers (as obtained by ground measurements or by extrapolation of meteorological analysis). A procedure has been set up which warns about these inconsistencies in near real time.
\end{abstract}

Key words GPS - double difference - zenith total delay - residuals

\section{Introduction}

Soon after its establishment twenty years ago, the Global Positioning System (GPS), became a new and powerful reference frame for active tracking of atmospheric phenomena. Microwave radio signals transmitted by 24 continuously operating GPS satellites are delayed by the atmospheric refraction as they propagate to Earth-based GPS receivers. GPS satellites transmit two $L$-band radio signals ( 19 and $22 \mathrm{~cm}$

Mailing address: Dr. Renato Santangelo, Dipartimento Ingegneria dei Materiali e dell' Ambiente (Osservatorio Geofisico), Università degli Studi di Modena e Reggio Emilia, Via Vignolese 905, 41100 Modena; e-mail: santangelo.renato@unimo.it wavelengths). Usually, the receiver's sampling rate is $30 \mathrm{~s}$ (observation epochs).

There is a dispersive effect of the ionosphere and a non-dispersive effect of the (neutral atmosphere) troposphere. Ionospheric effects could be largely removed by a linear combination of dual frequency data. When the ionosphere disturbance on GPS electromagnetic waves is resolved in a satisfactory manner, the main task remains to estimate better the neutral (basically tropospheric) atmosphere impact. The signal delay becomes a function of temperature, pressure and water content.

The wet component of the tropospheric delay in GPS signals is nearly proportional to the quantity of water vapor integrated along the signal path. Water vapor, as the only atmospheric constituent that possesses a permanent dipole moment (asymmetric distribution of charge in the water molecule) is a highly variable atmospheric constituent and also fundamental for the transfer of energy, producing major difficulties for the parameterization in Numerical 
Weather Prediction (NWP) modeling (Businger et al., 1996).

With the rapid growth in network of groundbased GPS receivers, such increased spatial and temporal resolution of the observing data enabled the birth of the new ground-based GPS meteorology which has expanded rapidly in recent years. The present state-of-the-art consists of currently advanced studies and certain operational services for monitoring of the atmospheric water vapor, climatology and weather analysis and prediction (Baker et al., 2001). The assimilated GPS observation data are used for the atmospheric modeling, primarily in parameter estimation.

Other sources of signal errors, such as ionospheric refraction, satellite orbit accuracy, antenna phase center modeling, signal multipath and scattering near the receiver, etc. have been significantly reduced during recent years (Bock and Doerflinger, 2001). Especially when the rather accurate near real time satellite orbits (with only $2 \mathrm{~h}$ time delay) became available recently, the space for the validation of numerical weather modeling was opened more widely. GPS meteorological estimates are now produced with accuracy comparable with that of existing meteorological measurement techniques (radiosondes, water vapor radiometers). Different efforts to use the elaboration of GPS specific tropospheric refraction as a meteorological profiles data source are being made today.

This paper aims to assimilate integrated meteorological measures obtained by GPS receivers with conventional meteorological measures in upper air like those provided by a meteorological database, e.g., the database of Deutscher WetterDienst (DWD) currently received in real time by Osservatorio Geofisico in Modena (Italy).

Meteorological fields, from the database, compute the corresponding integrated measure performed by GPS. A comparison shows possible inconsistencies, in near real time. The amount of detected inconsistencies (measured mean divided by standard deviation of the mean) represents a quality warning about the products (e.g., forecasts) emitted by such database. Obviously this quality warning concerns only possible errors of meteorological fields used in forecast models, without demanding that this quality warning also concern mechanisms used in the model.

\section{Summary of GPS activity within EEC project MAGIC (during MAP-SOP)}

The work uses GPS data observed in Padua and Medicina (near Bologna) during the Mesoscale Alpine Program-Special Observing Period (MAP-SOP - since September 1, 1999 up to November 30, 1999) (Bougeault et al., 2001). Two GPS receivers have been considered since they allow using a Double Difference Technique (better explained later) which increases precision of GPS analysis.

The receiver in Padua is used since it was close to the chosen MAP site in Verona, where about 100 balloons were launched and tracked on command of an MAP forecaster team in Innsbruck (Austria). The second receiver chosen was the one in Medicina (near Bologna), since balloons are also currently launched in Capofiume (near Bologna too) every day.

Upper air observations above Capofiume are currently received and assimilated by $\mathrm{Me}-$ teorological Centers (as e.g., DWD). Therefore their disseminated meteorological fields should represent fairly well meteorological conditions above Capofiume (i.e. above 1 or 2 GPS receivers) so that possible inconsistencies should be accounted to a lack of knowledge of meteorological conditions above the other GPS receivers (that in Padua).

\section{3. «Ionofree» combination of the two GPS signals emitted by a GPS satellite}

Each GPS satellite emits two signals, each having its own frequency. A suitable combination of such signals is called «ionofree» signal of that satellite. With a good approximation, the «ionofree» signal does not need any further correction during its travel across ionosphere. However, it undergoes refraction when it travels across the neutral atmosphere. Refraction depends on meteorological conditions along the traveled trajectory but does not depend on frequency (no dispersion) (Thayer, 1974). 
Obviously an in void «ionofree» signal would travel with the speed of light.

\section{GPS observable}

Due to a sophisticated procedure starting time $t_{I}$ of a GPS signal from a GPS satellite $I$, and arrival time $t_{\mathrm{A}}\left(>\mathrm{t}_{\mathrm{I}}\right)$ of the same GPS signal down to GPS receiver $A$, are appropriately recognized as belonging to the same signal. Travel time lag is measured by phase ranging: therefore, such time observable measured by phase ranging has an intrinsic phase ambiguity. Moreover, time lag or observable

$$
O=\left(t_{A}-t_{I}\right)
$$

has further errors due to unknown constants in both satellite and receiver clocks. In conclusion, observable $O$ should be written as

$$
O=\left[\left(t_{A}+k_{A}\right)-\left(t_{I}+k_{I}\right)\right]
$$

where $k_{A}$ and $k_{I}$ are unknown constants. Unknown constants disappear if a Double Difference of Observed phases is used i.e.

$$
\mathrm{DDO}=\left(O_{A}^{I}-O_{B}^{I}\right)-\left(O_{A}^{J}-O_{B}^{J}\right) .
$$

\section{Constraint if GPS signal travels in void}

An in void GPS signal would move with the speed of light $(=c)$, so that the following constraint exists

$$
c \cdot \mathrm{DDO}=\mathrm{DDL}
$$

where DDO means Double Difference of Observed phases (neglecting any problem concerning phase ambiguity) and DDL means Double Difference of Travel paths from satellites: $I, J$ to receivers $A, B$. At any epoch distances between receiver and satellite are fairly well known, due to rather well known orbits of satellites, under the assumption that the receiver does not move (no ocean loading). With selfexplanatory symbols

$$
\mathrm{DDL}=\left(L_{A}^{I}-L_{B}^{I}\right)-\left(L_{A}^{J}-L_{B}^{J}\right) .
$$

\section{GPS signal traveling across a neutral atmosphere}

When a GPS signal travels across a neutral atmosphere, i.e. a refractive atmosphere, it undergoes an extra-delay $T^{\prime}$, which exceeds the delay of a signal having the speed of light, due to a velocity lower than that of light. It is useful to convert extra-delay $T^{\prime}$ into a «tropospheric length» $T$

$$
T=c T^{\prime}
$$

Wave front of GPS signal which propagates towards Earth vertically along a line element $d s_{v}$ in neutral atmosphere, undergoes an extra-delay $d T_{v}$ which depends on meteorological parameters existing along that line element. Extra-delay (as length) from $I$ to corresponding vertical point $A_{v}$ on Earth is $T_{A_{v}}^{I}$.

Extra delay from a slant GPS satellite (say $I$ ) to a receiver (say $A$ ) is equal to extra delay from $I$ to point $A_{v}$ along vertical on the same wave front containing $A$, multiplied by a suitable mapping function, $f$

$$
T_{A}^{I}=T_{A_{v}}^{I} \cdot f .
$$

If zenith angle $z$ (complementary to elevation angle $e$ ) GPS satellite $I$ is less than $75^{\circ}$ (Duan et al., 1996), it can be assumed to be equal to

$$
f=1 / \cos (z) .
$$

Lack of knowledge of the exact expression of mapping function implies that an evaluation of $T$ is rather uncertain under slant conditions. For completeness, expression of DDT is shown

$$
\mathrm{DDT}=\left(T_{A}^{I}-T_{B}^{I}\right)-\left(T_{A}^{J}-T_{B}^{J}\right) .
$$

\section{Constraint in neutral atmosphere}

Therefore previous constraint (in Double Difference Technique) must be suitably modified into the following:

$$
c \cdot \mathrm{DDO}=\mathrm{DDL}+\mathrm{DDT} .
$$



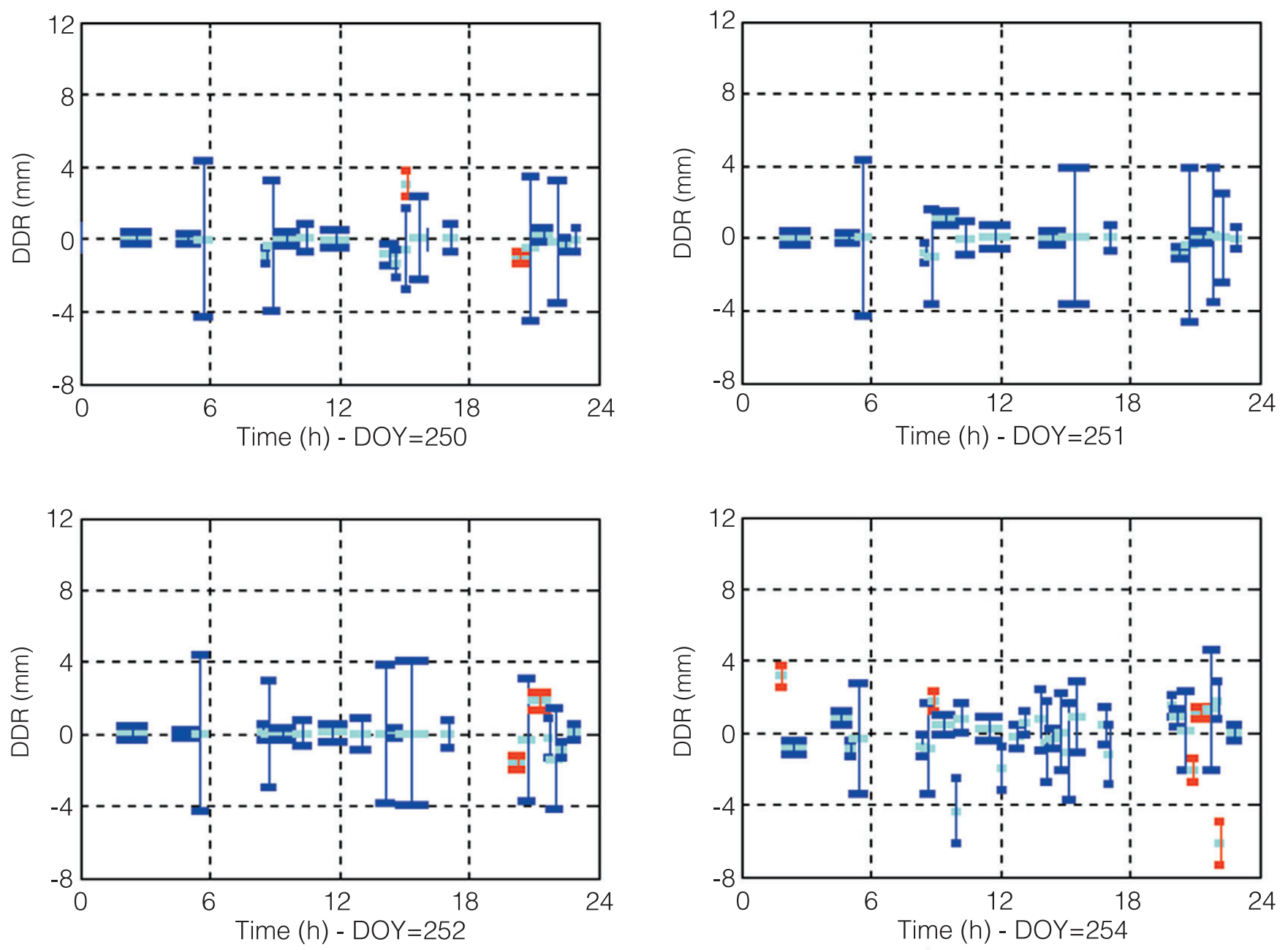

Fig. 1. Double differences residuals versus time for DOY: 250, 251, 252 and 254 of the year 1999 for the GPS stations Padua and Medicina (Italy). Lines: cyan-mean DDR; blue-standard deviation; red-anomalous period.

DDO is obtained by phase ranging; DDL is obtained by known orbits of satellites. DDT can be obtained as follows:

1) by meteorological database disseminated by Meteorological Centers (e.g., by DWD) and received in real time;

2) by ground meteorological measurements in real time or by climatological data, suitably extrapolated towards upper air, e.g., by a formula suggested by Saastamoinen (1972).

These two possible choices for evaluation of extra-delay DDT are foreseen in the «Bernese» software (to be discussed in next section). The two choices provide, for most epochs, close results.

\section{The «Bernese GPS Software» application for a quality index epoch by epoch}

Previous three terms in eq. (7.1) are adjusted by «Bernese» software (Hugentobler et al., 2001) by a suitable fit which takes into account phase ambiguity also, and provides finally an unbalance or a Double Difference Residual (DDR).

If the fit were perfect DDR would be zero. Upper air meteorology, analyzed by the «Bernese» software, during MAP-SOP, was provided by:

1) measurements at ground and suitably extrapolated to upper air;

2) meteorological database disseminated by DWD. 

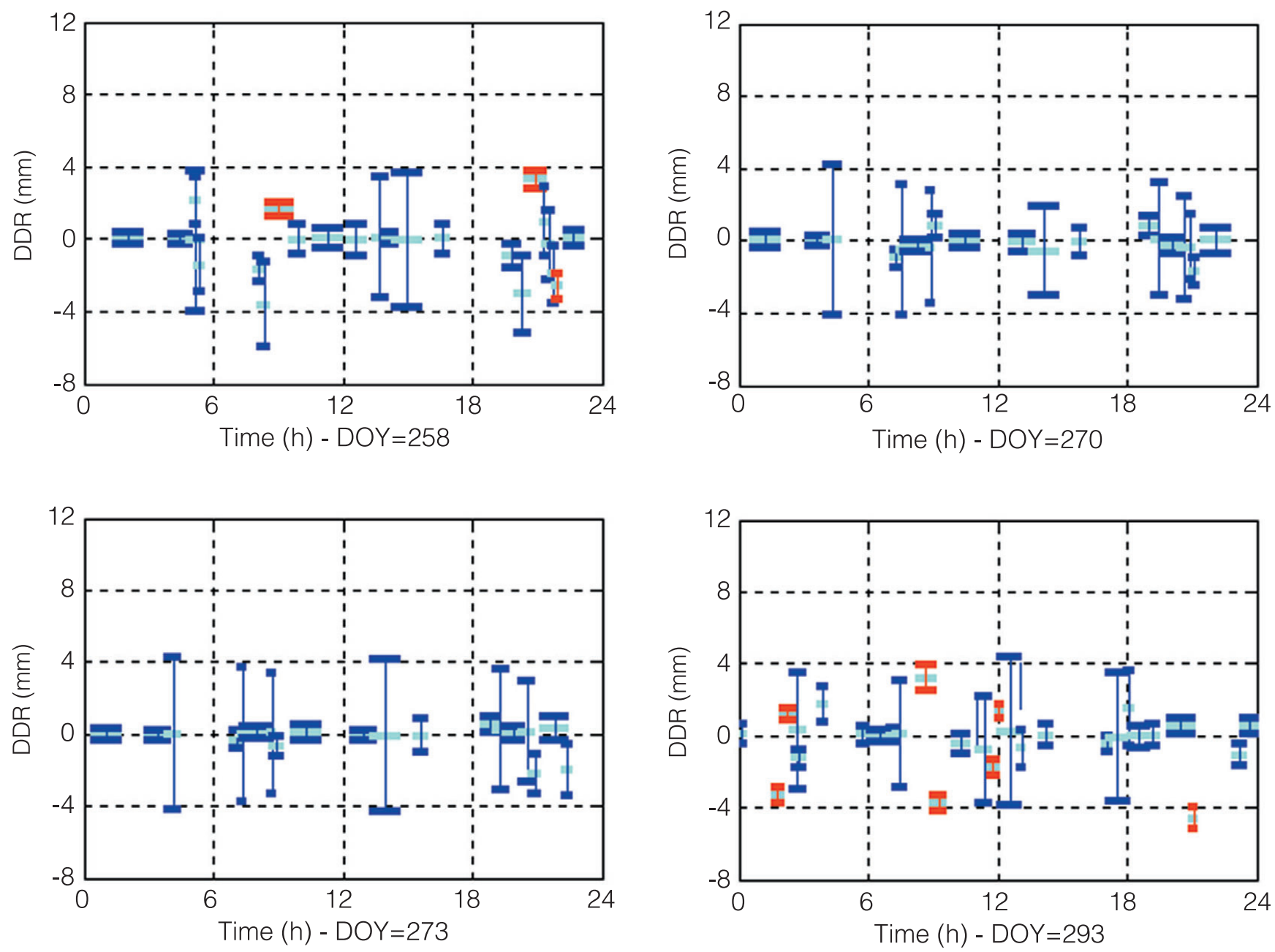

Fig. 2. Double differences residuals versus time for DOY: 258, 270, 273 and 293 of the year 1999 for the GPS stations Padua and Medicina (Italy). Lines: cyan-mean DDR; blue-standard deviation; red-anomalous period.

It seems preferable to use meteorological fields provided by DWD database since they are expected to represent better upper air conditions.

Total number of 13 days, belonging to the MAP time series of observations have been analyzed so that their DDR is available versus time.

\section{Pairs of satellites observing a pair of receivers at each epoch: zenith cut-off}

At any epoch there are several satellites which observe a pair of receivers in sites $A$ and $B$. Therefore there are several pairs of satellites, which provide different values of DDR. In order to avoid management of uncertain DDRs, due to uncertain mapping functions, it would be preferable to choose near vertically positioned satellites or small zenith angles. However, with such choice, only very few satellites would be accepted. Therefore a compromise is necessary.

Zenith angles smaller than $30^{\circ}$, for each pair of satellites, observing the two receivers in $A$ and $B$, seem a reasonable compromise. Certainly this choice of a cut-off angle implies a significant reduction of pairs of satellites observing simultaneously receivers $A, B$ at that epoch.

With such choice, at any day, not more than two pairs of satellites are simultaneously present. 

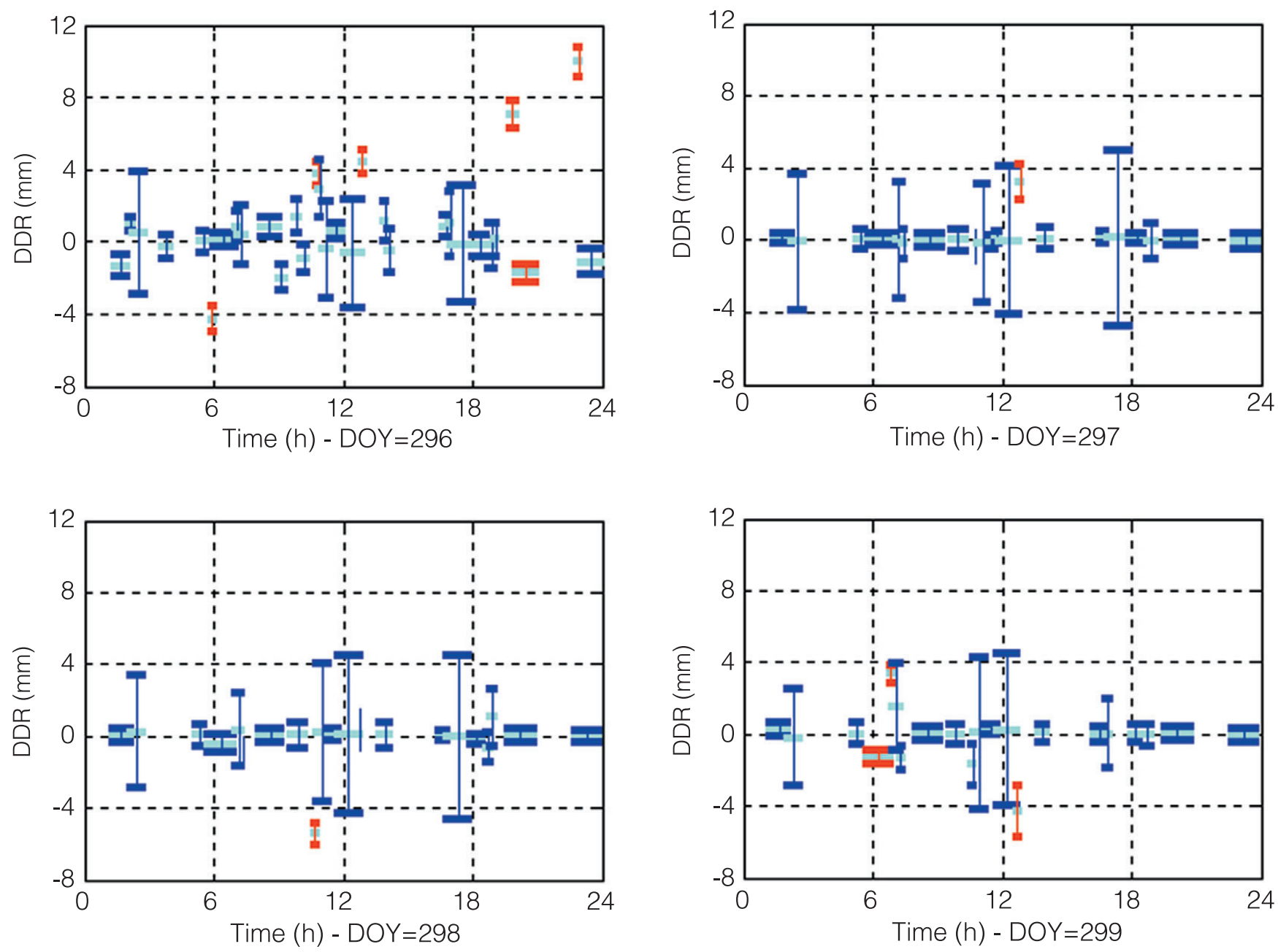

Fig. 3. Double differences residuals versus time for DOY: 296, 297, 298 and 299 of the year 1999 for the GPS stations Padua and Medicina (Italy). Lines: cyan-mean DDR; blue-standard deviation; red-anomalous period.

\section{Evolution of DDR versus epoch; periods of consecutive epochs}

Since DDR fitted by the procedure previously outlined in eq. (6.1) fluctuates, it is better to consider an average over several consecutive epochs during which the satellite constellation remain unchanged. In fact, reception of a same pair of satellites lasts several epochs (more than 10 epochs, are chosen, i.e. $>5 \mathrm{~min}$ ). During that period, every DDR should vanish, if the fit was ideal. Therefore it is preferable to consider the mean value of DDR: $\overline{\mathrm{DDR}}$, and the standard deviation of the mean: $\sigma_{\overline{\mathrm{DDR}}}$, for that period.
For all 13 days previously mentioned, GPS was observed continuously, and meteorological data from DWD database were obtained. In figs. 1 to 4 , evolution of $\overline{\mathrm{DDR}}$ and $\sigma_{\overline{\mathrm{DDR}}}$ is shown for 13 days with suitable colors (DOY represents Day Of Year, e.g. DOY 250 is September 07th that year) for every period of consecutive epochs with the same satellites observing. In the same plot, anomalous periods are suitably labeled in red. To determine an anomalous period, a hypothesis test was done. Taking a significance level of $99.7 \%$, anomalous periods of $\overline{\mathrm{DDR}}$ happen when $\overline{\mathrm{DDR}}$ differs from its expected value (zero) by more than 3 standard deviations of the mean i.e.: $-3 \sigma_{\overline{\mathrm{DDR}}} \leq \overline{\mathrm{DDR}} \leq 3 \sigma_{\overline{\mathrm{DDR}}}$. 


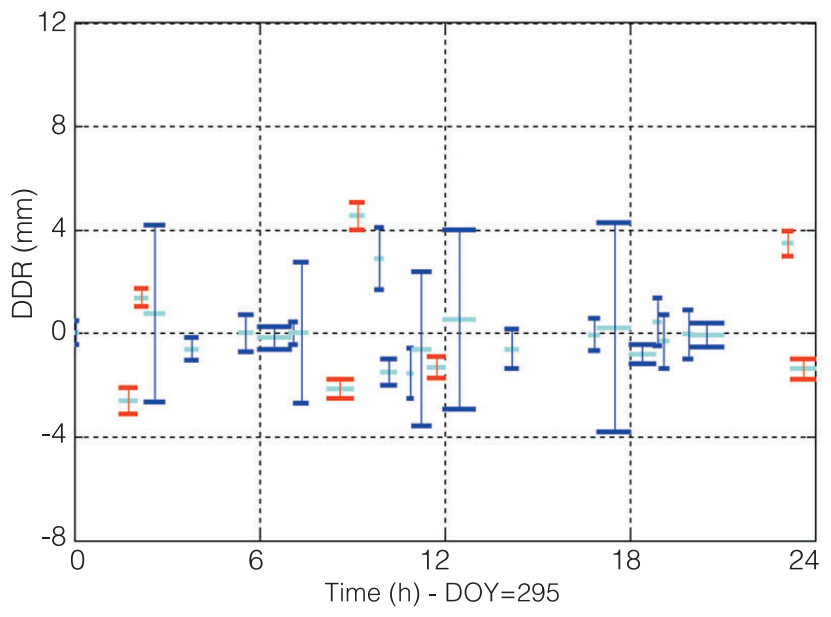

Fig. 4. Double differences residuals versus time for DOY: 295 of the year 1999 for the GPS stations Padua and Medicina (Italy). Lines: cyan - mean DDR; bluestandard deviation; red-anomalous period.

\section{Anomalous periods}

Anomalous periods indicate that a certain inconsistency exists between measured data (DDO from GPS), orbital data (DDL), and meteorological fields (DDT). The latter are obtained by ground measurements aside receiver or by extrapolation of DWD analysis.

\section{Conclusions}

A procedure has been set up which, in near real time, warns about consecutive epochs with the same satellites, which show inconsistencies among all available information.

However, the procedure needs, simultaneous observations of two satellites and two receivers. These inconsistencies may account for a lack of precise knowledge of meteorological information above at least one of the two receivers. The ambiguous assignment of the imprecise meteorological condition is the main drawback of procedure. However, suitable additional meteorological measurements close to one of the two receivers may help in defining which receiver site is the imprecise one.

\section{REFERENCES}

BAKer, H.C., A.H. Dodson, N.T. Penna, M. Higgins and D. OFFILER (2001): Ground-based GPS water vapour estimation: potential for meteorological forecasting, J. Atmos. Solar-Terr. Phys., 63, 1305-1314.

Bock, O. and E. Doerflinger (2001): Atmospheric modeling in GPS data analysis for high accuracy positioning, Phys. Chem. Earth (A), 26 (6-8), 373-383.

Bougeault, P., P. Binder, A. BuZzi, R. DiRKs, R. Houze, J. Kuettner, R.B. Smith, R. Steinacker and H. VOLKERT (2001): The MAP special observing period, Bull. Am. Meteorol. Soc., 82, 637-650.

Businger, S., S.R. Chiswell, M. Bevis, J. Duan, R.A. Anthes, C. Rocken, R.H. WARE, M. Exner, T. VANHOVE and F.S. SOLHEIM (1996): The promise of GPS in atmospheric monitoring, Bull. Am. Meteorol. Soc., 77, 5-18.

Duan, J., M. Bevis, P. FAng, Y. Bock, S. Chiswell, S. Businger, C. Rocken, F. SOlHeim, T. VAN HoOve, R. WARE, S. MCClusky, T.A. HERRING and R.W. KING (1996): GPS meteorology: direct estimation of the absolute value of precipitable water, J. Appl. Meteorol., 35, 830-838.

HugENTOBLER, U., S. SCHAER and P. FRIDEZ (Editors) (2001): Bernese GPS Software, Version 4.2, Astronomical Institute, University of Berne.

SAASTAMOINEN, J. (1972): Atmospheric correction for the troposphere and stratosphere in radio ranging of satellites, in The Use of Artificial Satellites for Geodesy, Geophys. Monogr. Ser., edited by S.W. HENRIKSEN, A. MANCINI and B.H. CHOVITZ (AGU, Washington D.C.), 15, 245-251.

THAYER, D. (1974): An improved equation for the radio refractive index of air, Radio Sci., 9, 803-807 